\title{
The clinical impact of three validated PD-L1 immunohistochemistry assays as a prognostic factor in small cell lung cancer
}

\author{
Yong Seok Lee ${ }^{1 \#}$, Jun Hyeok Lim ${ }^{2 \#}$, Wookyung Ryu ${ }^{2}$, Mi Hwa Park², Lucia Kim ${ }^{3}$ Kang Kim , \\ Woo Youl $\mathrm{Kim}^{4}$, Hae-Seong $\mathrm{Nam}^{2} \wedge$ \\ ${ }^{1}$ Department of Obstetrics and Gynecology, College of Medicine, The Catholic University of Korea, Seoul, Korea; ${ }^{2}$ Division of Pulmonology, \\ Department of Internal Medicine, Inha University Hospital, Inha University School of Medicine, Incheon, Korea; ${ }^{3}$ Department of Pathology, Inha \\ University Hospital, Inha University School of Medicine, Incheon, Korea; ${ }^{4}$ Division of Pulmonary and Critical Care Medicine, Department of \\ Internal Medicine, Incheonsarang Hospital, Incheon, Korea \\ Contributions: (I) Conception and design: YS Lee, HS Nam; (II) Administrative support: HS Nam; (III) Provision of study materials or patients: JH \\ Lim, L Kim, K Kim, WY Kim, HS Nam; (IV) Collection and assembly of data: All authors; (V) Data analysis and interpretation: YS Lee, JH Lim, L \\ Kim, HS Nam; (VI) Manuscript writing: All authors; (VII) Final approval of manuscript: All authors. \\ \#These authors contributed equally to this work. \\ Correspondence to: Hae-Seong Nam, MD, PhD. Division of Pulmonology, Department of Internal Medicine, Inha University Hospital, Inha \\ University School of Medicine, Incheon, 22332, Korea. Email: hsnam@inha.ac.kr.
}

Background: Evidence of the clinical impact of programmed death-ligand 1 (PD-L1) expression in small cell lung cancer (SCLC) is scarce and conflicting, even though atezolizumab became the first PD-L1 inhibitor approved by the US Food and Drug Administration (FDA) in recent years for the initial treatment of extensive-stage (ES)-SCLC.

Methods: We investigated PD-L1 expression in SCLC tumors using the three validated PD-L1 immunohistochemistry (IHC) assays (SP263, SP142, and 22C3) and assessed the correlation between PD-L1 expression and clinicopathological factors to determine the prognostic value of PD-L1 expression. The three PD-L1 IHC analyses were prospectively used to assess tumor samples of patients with SCLC at diagnosis.

Results: Of the total of 59 patients, 47 patients received the active treatment beyond platinum-based chemotherapy at our institution. PD-L1 expression was positive in 39.0\% with SP263, 37.3\% with SP142, and $22.0 \%$ with $22 \mathrm{C} 3$. In a univariate analysis, the positive result of at least one of the three PD-L1 assays and the positive result of the SP142 assay were associated with longer overall survival (OS). A multivariable analysis confirmed that performance status, stage, and the SP142 assay were independent predictors of OS. In subgroup analysis, these results revealed more significant prognostic factors in ES than in limited-stage (LS). In patients with SCLC, especially those with ES, the expression of the SP142 assay is a significant independent prognostic factor.

Conclusions: Although these results need to be further validated in larger cohorts, this information will benefit clinicians and patients in determining the immunotherapy for patients with ES-SCLC.

Keywords: Immunotherapy; prognostic factor; programmed death-ligand 1 (PD-L1) assay; small cell lung cancer (SCLC); SP142

Submitted Mar 02, 2021. Accepted for publication May 05, 2021.

doi: $10.21037 /$ tlcr-21-165

View this article at: http://dx.doi.org/10.21037/tlcr-21-165

\footnotetext{
$\wedge$ ORCID: 0000-0003-2543-6612.
} 


\section{Introduction}

Small cell lung cancer (SCLC) globally accounts for 13\% of all new lung cancers. It is a highly aggressive tumor that originates from the precursors of neuroendocrine cells, characterized by a high proliferation rate and early metastasis. Although SCLC is initially very sensitive to conventional treatments such as platinum-doublet-based chemotherapy and/or radiotherapy, it has been observed to develop early resistance to such treatments in most cases; therefore, it is considered a recalcitrant neoplasm with a 5 -year survival rate of less than $6 \%$ (1). Unfortunately, these conventional treatment strategies for SCLC have remained at a plateau over the past three decades.

The clinical development of immunotherapy using immune checkpoint inhibitors (ICIs) is a revolutionary milestone in the field of several cancer treatments, including lung cancer $(2,3)$. ICIs targeting the signaling of programmed death 1 (PD-1) or its ligand 1 (PD-L1) have shown promising responses in some patients with advanced non-small cell lung cancer (NSCLC) (4-6). However, the clinical response rate of $\mathrm{PD}-1 / \mathrm{PD}-\mathrm{L} 1$ inhibitors against several solid tumors, including lung cancer, is only approximately $20 \%(4,6,7)$. Thus, predictive biomarkers represnet an unmet need to identify patients who may clinically benefit from PD-1/PD-L1 inhibitors. Currently, four immunohistochemistry (IHC) assays, namely PD-L1 SP263 and SP142 (VENTANA), and PDL1 IHC 22C3 and 28-8 (pharmDx DAKO), have been registered by the US Food and Drug Administration (FDA) as companion and complementary diagnostic assays for detecting the expression of PD-L1 to enrich the patient response to $\mathrm{PD}-1 / \mathrm{PD}-\mathrm{L} 1$ inhibitors in clinical practice $(8,9)$. Although PD-L1 expression on tumor cells (TC) or immune cells (IC) in NSCLC, which reflect immune-active microenvironments, has been recognized as a predictive biomarker of a clinical response to PD-1/PD-L1 inhibitors $(10-12)$, their correlation is not absolute $(5,6,13)$. On the other hand, PD-L1 expression is associated with poor prognosis in several solid tumors, included NSCLC (14-16).

Recently, a clinical study reported good outcomes for combination therapy with PD-L1 inhibitor and conventional chemotherapy in patients with extensivestage (ES)-SCLC (17). Based on findings of this study, atezolizumab became the first PD-L1 inhibitor approved by the FDA for the initial treatment of ES-SCLC. However, evidence of clinical impact as a predictive and/or prognostic biomarker of PD-L1 expression in SCLC is scarce and conflicting. Recent meta-analyses of clinical impact of PDL1 expression in SCLC revealed inconsistent results $(18,19)$. Thus, the association of PD-L1 expression in SCLC with clinicopathological factors warrants further investigation. For this reason, we investigated PD-L1 expression in SCLC tumors using the three validated PD-L1 IHC assays (SP263, SP142 and 22C3), which are the most widely used in clinical practice, and assessed the correlation between PDL1 expression and clinicopathological factors to determine its prognostic value. We present the following article in accordance with the REMARK reporting checklist (available at http://dx.doi.org/10.21037/tlcr-21-165).

\section{Methods}

\section{Study design and patients}

From December 2017 to February 2020 at Inha University Hospital, patients who were histologically confirmed to have primary SCLC and had sufficient tissue for three different PD-L1 IHC staining were considered eligible for the study. Patients diagnosed with other cancers within the previous 5 years were excluded from the study. Baseline prognostic clinicopathological and laboratory variables were collected retrospectively from the electronic medical record (EMR) system. Patient-related variables included age, gender, smoking status, Eastern Cooperative Oncology Group performance status (ECOG PS), and the serum levels of lactate dehydrogenase (LDH), carcinoembryonic antigen (CEA), and neutrophil/lymphocyte ratio (NLR) at diagnosis. The tumor-related variables consisted of histology and stage. All patients were staged using both the Veterans Administration and the $8^{\text {th }}$ edition of the TNM classification system (20) based on contrast chest computed tomography (CT) (and/or abdomen CT), brain magnetic resonance imaging (and/or brain $\mathrm{CT}$ ), and positron emission tomography (PET)/CT (and/or whole-body bone scan) at the time of diagnosis.

Written informed consent for testing PD-L1 IHC assays was obtained from all patients before they underwent the tissue biopsy for lung cancer diagnosis. This study was conducted in accordance with the provisions of the Declaration of Helsinki (as revised in 2013) and was approved by the Institutional Review Board of Inha University Hospital (No. 202008032). Survival data were collected from the EMR system and the Korean Ministry of 
Security and Public Administration.

\section{PD-L1 IHC}

PD-L1 expression was assessed in formalin-fixed paraffin embedded tumor samples obtained by tissue biopsy from each patient at diagnosis. The three most widely used validated PD-L1 IHC assays, namely VENTANA PD-L1 SP263 and SP142 (Ventana Medical Systems, Inc., Tucson, AZ, USA), and PD-L1 IHC 22C3 pharmDx (Agilent Technologies/Dako, Carpinteria, CA, USA), were used on the same tissue. Assays with SP142 were performed with the BenchMark XT staining instrument (Ventana Medical Systems) according to the protocols included in the instructions for use of the antibodies and the external quality control system from Nordic IHC Quality Control (NordiQC) for SP263. We detected antibody staining with an OptiView DAB IHC Detection Kit with (for the SP142 antibody) and without (for the SP263 antibody) the OptiView Amplification Kit (Ventana Medical Systems), in accordance with the protocols recommended by the manufacturer $(8,21)$. The pharmDx $22 \mathrm{C} 3$ was tested with Dako Autostainer Link 48 (Agilent) using the optimized closed protocol provided by the manufacturer for the automated platform $(13,21)$. All stained slides were evaluated by a board-certified pathologist who was blinded to the patients' clinical information according to the scoring protocol of each system. The tumor proportion score (TPS) or TC and tumor-infiltrating IC were calculated as the percentage of $\mathrm{PD}-\mathrm{L} 1$ positive TC and IC with membrane and cytoplasmic staining of any intensity, respectively. In these assays, the TPS was applied for $22 \mathrm{C} 3$ and SP263, and TC and IC were applied for SP142. Positivity of 22C3 and SP263 was defined as PD-L1 expression $\geq 1 \%$ of TPS, and positivity of SP142 was defined as PD-L1 expression $\geq 1 \%$ of either TC or IC.

\section{Statistical analysis}

The distribution of clinicopathological and laboratory variables according to the positive result for at least one of the three PD-L1 assays (1/3 PD-L1 assays) was assessed using the chi-square test or Fisher's exact test for categorical values. Cutoff values of CEA and LDH were determined by the normal reference range in our institutions. The optimal cutoff value of NLR was determined by receiver operating characteristic (ROC) curve analysis as the points at which the Yonden index (sensitivity + specificity -1 ) values were maximal, using overall survival (OS) as the endpoint. The correlation and agreement between assays were examined by Spearman's rank correlation coefficient (rho) and Cohen's kappa coefficient ( $\kappa)$. OS was defined as the time interval from the date of diagnosis to the date of death or the last follow-up. Survival analyses were performed using the Kaplan-Meier method and log-rank test. Potential predictors of survival were entered into univariate KaplanMeier models and compared using the log-rank test. Factors with a prognostic association in the univariate analysis were entered into a multivariate Cox regression model to determine their independent effects. Variables selection method for Cox regression models was used the forward sequential method, which has been widely used for smaller models $(22,23)$. The results of Cox regression modeling were presented as hazard ratios and their associated $95 \%$ confidence intervals (CI). All analyses were performed using SPSS version 26.0 (SPSS, Chicago, IL, USA). Statistical significance was defined as a two-sided $\mathrm{P}<0.05$.

\section{Results}

\section{Patient characteristics}

A total of 59 SCLC patients were included in the analysis. The tissue specimens for evaluating three PD-L1 assays were obtained via bronchoscopic biopsy $(n=34)$, CTguided transthoracic needle biopsy $(\mathrm{n}=13)$, tissue cores via endobronchial ultrasound-transbronchial needle aspiration of a mediastinal lymph node ( $\mathrm{n}=9$ ), biopsy of the supraclavicular lymph node $(n=2)$, and pleural biopsy $(n=1)$. The baseline characteristics of the study populations are summarized in Table 1. The median age of the patients was 67 years (range, 50-95), and there were 53 (89.8\%) males. The majority of patients were former or current smokers (94.9\%) and had an ECOG PS of 0-1 (81.4\%). Thirty patients (50.8\%) had limited-stage (LS) and 29 (49.2\%) had ES at the time of diagnosis. The median serum NLR, LDH, and CEA levels were 3.0 (range, 0.9-24.8), 265 IU/L (range, 128-1,709), and $7.63 \mathrm{ng} / \mathrm{mL}$ (range, 0.93-405), respectively. The treatment modalities were determined based on the patient's PS and the opinions of the patient and his/her family. Seventeen patients of LS received concurrent platinum-etoposide (PE) chemo and radiation therapy, 2 patients of LS received lobectomy and/or PE chemotherapy, 2 patients of LS received PE chemo and sequential chest radiation therapy, 6 patients of LS and 20 patients of ES received PE chemotherapy, one patient 
Table 1 Baseline characteristics according to positive results for at least one of the three PD-L1 assays

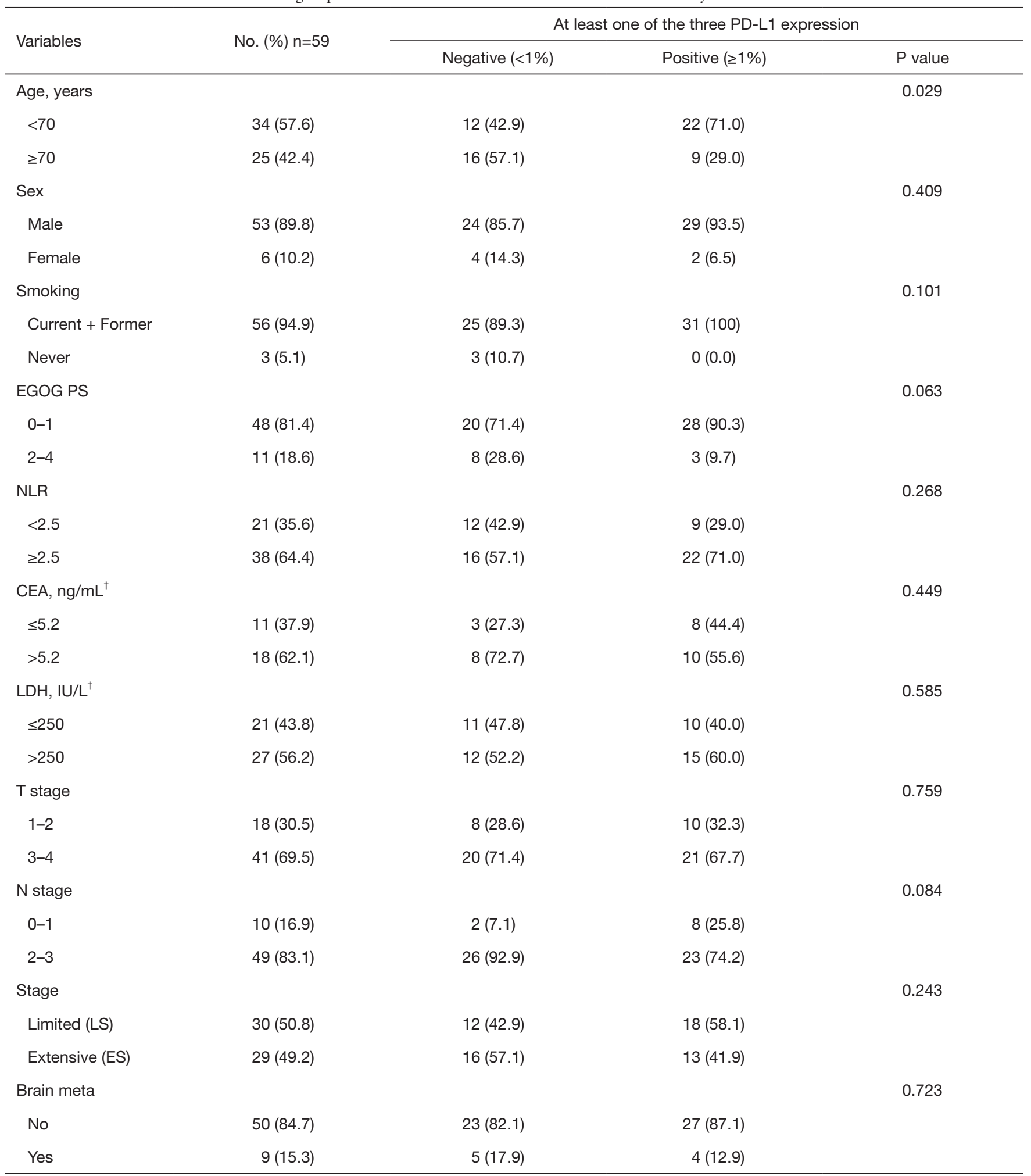

Table 1 (continued) 
Table 1 (continued)

\begin{tabular}{|c|c|c|c|c|}
\hline Variables & No. $(\%) n=59$ & \multicolumn{3}{|c|}{ At least one of the three PD-L1 expression } \\
\hline SP263 & & & & $<0.001$ \\
\hline Negative & $36(61.0)$ & $28(100)$ & $8(25.8)$ & \\
\hline Positive & $23(39.0)$ & $0(0.0)$ & $23(74.2)$ & \\
\hline Negative & $37(62.7)$ & $28(100)$ & $9(29.0)$ & \\
\hline Positive & $22(37.3)$ & $0(0.0)$ & $22(71.0)$ & \\
\hline $22 \mathrm{C} 3$ & & & & $<0.001$ \\
\hline Negative & $46(78.0)$ & $28(100)$ & $18(58.1)$ & \\
\hline
\end{tabular}

${ }^{\dagger}$, dichotomized by cutoff of normal value. CEA, carcinoembryonic antigen; ECOG PS, Eastern Cooperative Oncology Group performance status; LDH, lactate dehydrogenase, NLR, neutrophil-to-lymphocyte ratio.
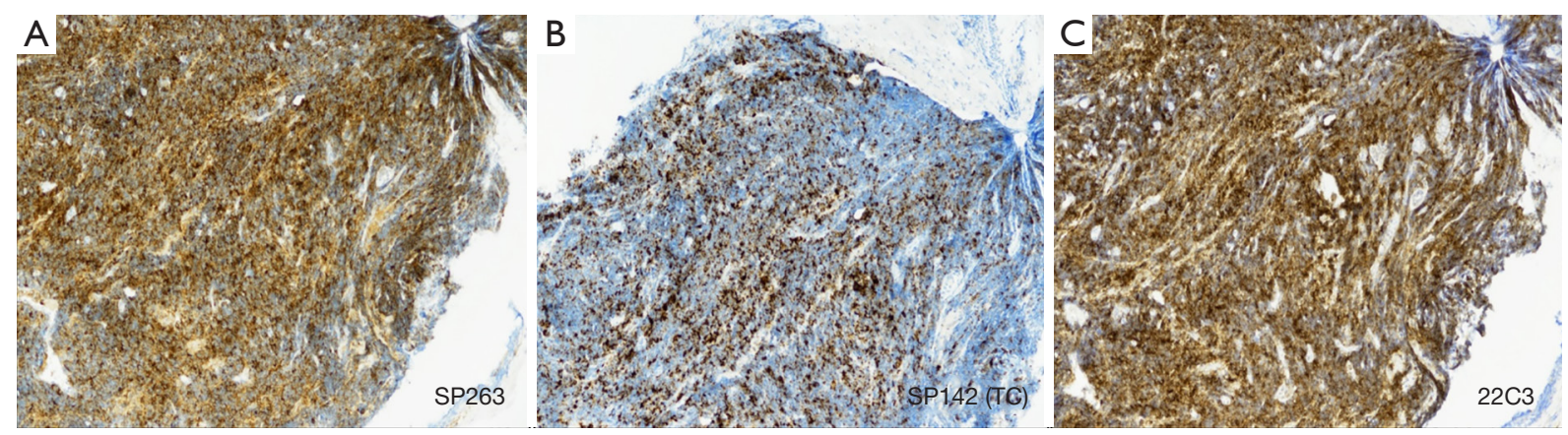

Figure 1 Representative positive images of the same small cell lung cancer (SCLC) sample stained with three different PD-L1 assays ( $\times 100)$ : (A) VENTANA PD-L1 SP263 (30\%), (B) VENTANA PD-L1 SP142 (20\%), and (C) PD-L1 IHC 22C3 pharmDx (30\%) assays showed strong PD-L1 positivity in tumor cells (TC).

of LS and one patient of ES received only chest radiation therapy, and the other 10 patients received supportive care or chose to go to another hospital.

\section{Prevalence and correlation of PD-L1 expression}

Figure 1 illustrates the positive images of staining with three different PD-L1 assays in a representative SCLC sample. PD-L1 expression was positive in 23 cases $(39.0 \%)$ with SP263, 22 cases $(37.3 \%)$ with SP142 [19 cases $(32.2 \%)$ on IC and 5 cases $(8.5 \%)$ on TC), and 13 cases (22.0\%) with 22C3]. Thirty-one patients with SCLC (52.5\%) were positive for 1/3 PD-L1 assays. Nine patients (15.3\%) were positive for all three PD-L1 assays, and 28 patients
(47.5\%) were negative for all three PD-L1 assays (Figure 2). Although the different assays are approved or in development as companion or complementary diagnostics to different ICIs agents, targeting of PD-1/PD-L1 have the same pathway (8). In this study, we assigned two categories according to positivity for $1 / 3$ PD-L1 assays. The relation between positivity for 1/3 PD-L1 assays and the patient's clinicopathological variables is shown in Table 1. Positivity for 1/3 PD-L1 assays was significantly correlated with age $(\mathrm{P}=0.029)$. No significant correlation was observed between the positive for $1 / 3 \mathrm{PD}-\mathrm{L} 1$ assays and the other factors, including sex, smoking status, ECOG PS, stage, NLR, CEA, and LDH. The analysis of each PD-L1 assay also showed similar results (Table S1). 


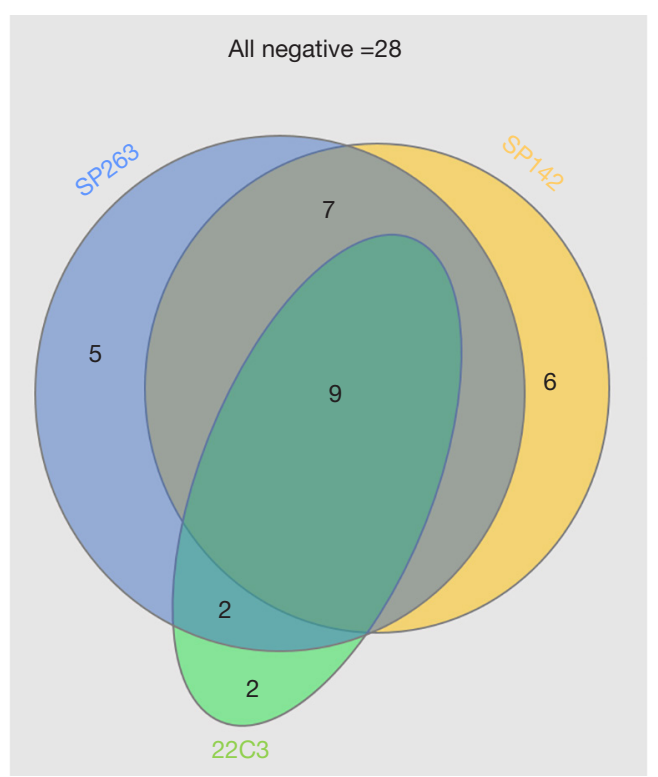

Figure 2 Venn diagram showing PD-L1 immunochemistry analysis using the three PD-L1 assays in 59 patients with small cell lung cancer (SCLC).
Further analysis was performed to assess the correlation between the different PD-L1 assays. The correlation coefficients (rho) were $0.550(\mathrm{P}<0.001)$ and $0.551(\mathrm{P}<0.001)$ between SP263/SP142 assays and SP263/22C3 assays, respectively (Figure S1A,B). The concordance rates of these assays (SP263/SP142 assays and SP263/22C3 assays) were $78 \%$ with $\kappa=0.533(\mathrm{P}<0.001)$ and $76.3 \%$ with $\kappa=0.459$ $(\mathrm{P}<0.001)$, respectively. The rho was $0.365(\mathrm{P}=0.004)$ and the concordance rate was $71.2 \%(\kappa=0.328, \mathrm{P}=0.007)$ between SP142/22C3 assays (Figure S1C). These results indicate that the SP263 assay and the other two assays had a moderate to strong correlation with a very high statistical significance $(\mathrm{P}<0.001)$, whereas SP142 and $22 \mathrm{C} 3$ has a weak to moderate correlation (24).

\section{Three PD-L1 expression and OS}

The median survival time (MST) of all patients was 398 days (95\% CI: 262-534 days). The results of the univariate analyses of individual baseline variables are listed in Table 2 .

Table 2 Univariate and multivariate analyses of the factors that are predictive of overall survival in all patients $(\mathrm{n}=59)$

\begin{tabular}{|c|c|c|c|c|c|c|}
\hline Variable & \multicolumn{3}{|c|}{ Univariate analysis } & \multicolumn{3}{|c|}{ Multivariate analysis } \\
\hline Age, years & & & 0.004 & & & \\
\hline$<70$ & 453 & $277-629$ & & & & \\
\hline$\geq 75$ & 201 & $178-224$ & & & & \\
\hline Male & 398 & $259-537$ & & & & \\
\hline Female & 414 & $105-723$ & & & & \\
\hline Smoking habit & & & 0.555 & & & \\
\hline Current + Former & 414 & $364-464$ & & & & \\
\hline $0-1$ & 435 & $356-514$ & & Reference & & \\
\hline$\geq 2$ & 69 & $0-138$ & & 4.83 & $1.92-12.1$ & \\
\hline NLR & & & 0.03 & & & \\
\hline$<2.5$ & 575 & $320-830$ & & & & \\
\hline$\geq 2.5$ & 242 & $117-367$ & & & & \\
\hline
\end{tabular}

Table 2 (continued) 
Table 2 (continued)

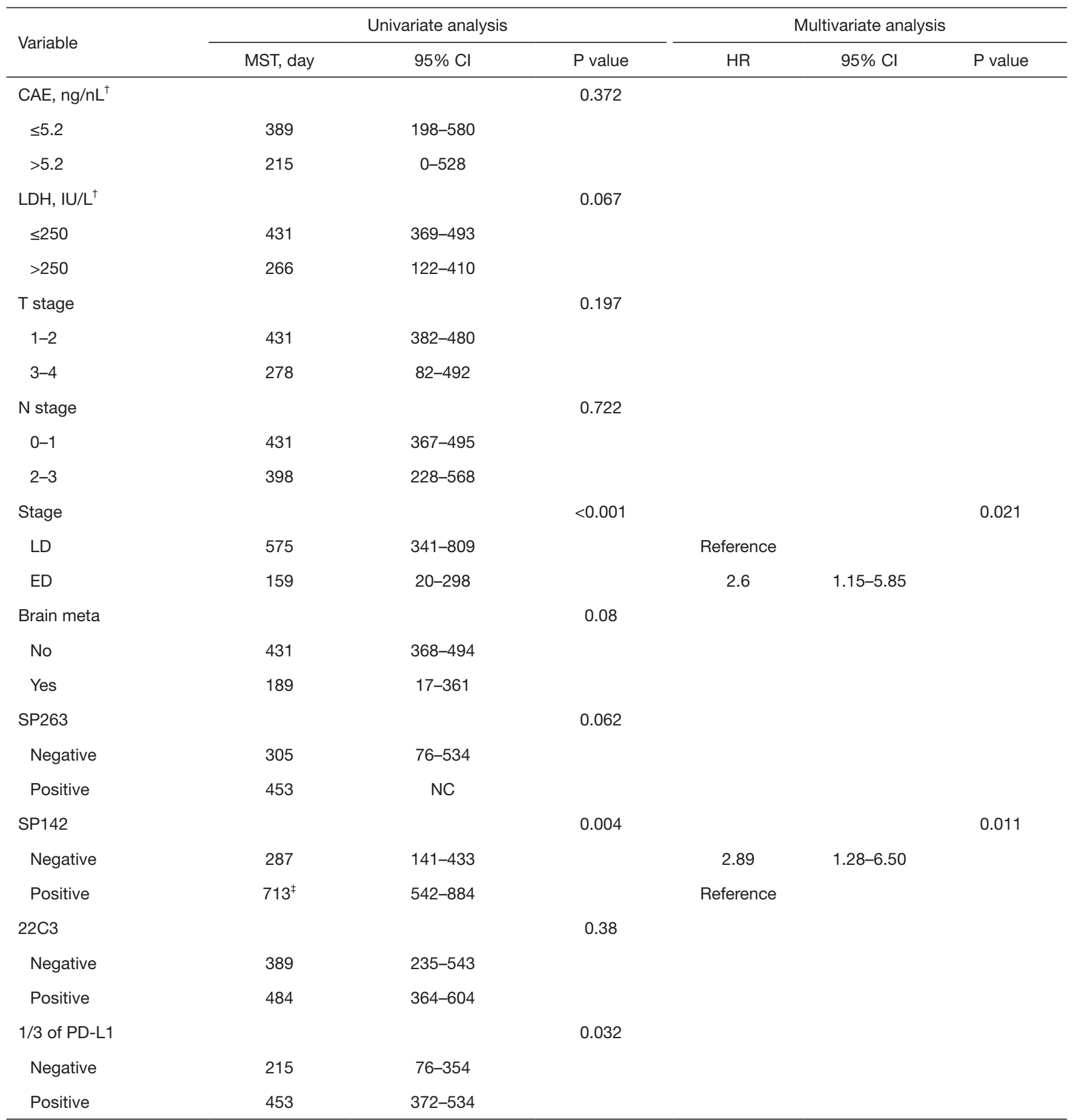

${ }^{\dagger}$, dichotomized by cutoff of normal value. ${ }^{\ddagger}$, the mean survival time is used because the median suvival time is not calculated. CEA, carcinoembryonic antigen; Cl, confidence interval; ECOG PS, Eastern Cooperative Oncology Group performance status; HR, hazard ratio; LDH, lactate dehydrogenase; MST, median survival time, NC, not calculated; NLR, neutrophil/lymphocyte ratio; 1/3 of PD-L1, at least one of the three PD-L1 assays. 

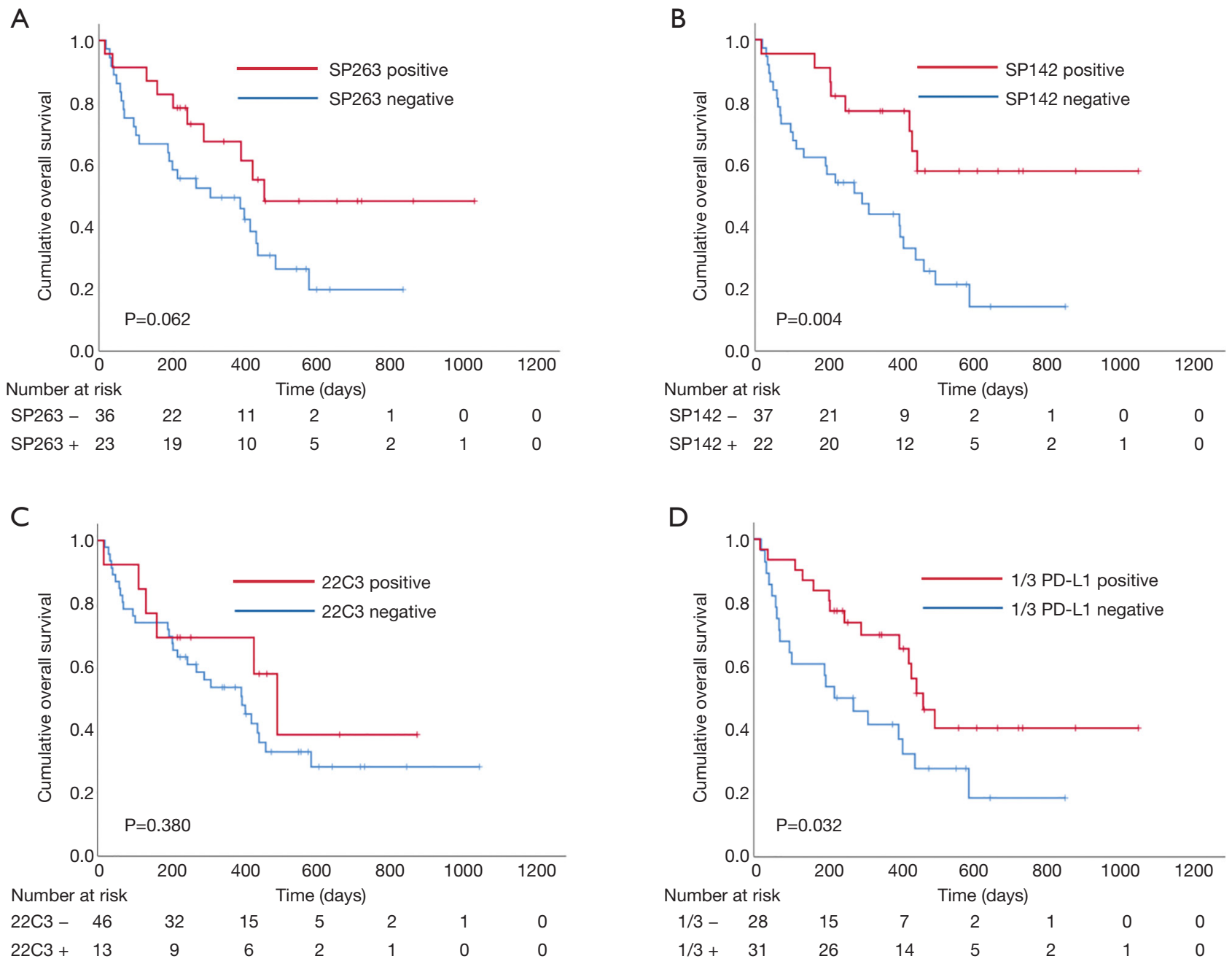

Figure 3 Kaplan-Meier curves of overall survival according to PD-L1 expression status in small cell lung cancer (SCLC) patients (n=59): (A) PD-L1 SP263, (B) PD-L1 SP142, (C) PD-L1 IHC 22C3, (D) positive results for at least one of the three PD-L1 assays (1/3 PD-L1).

The positive group for 1/3 PD-L1 assays had significant prognostic factors in the univariate analysis (MST $453 \mathrm{vs.}$ 215 days, $\mathrm{P}=0.032)$. The positive group of each $\mathrm{PD}-\mathrm{L} 1$ assay showed longer OS than the negative group of each PD-L1 assay, but there was only a significant difference in the SP142 assay $(\mathrm{P}=0.004)$ (Figure 3). The following variables were associated with longer OS: age $<70(\mathrm{P}=0.004)$, ECOG PS $0-1(\mathrm{P}<0.001)$, stage $(\mathrm{P}<0.001)$, and NLR $<2.5$ $(\mathrm{P}=0.030)$. In subgroup analysis of ES ( $\mathrm{n}=29)$, the positive group of each PD-L1 assay had longer OS with more distinct differences than with the survival analysis of LS patients (Figure S2). Individual variables that were analyzed in the univariate analyses were entered into the multivariate Cox model, irrespective of their significance. A multivariate analysis revealed the following prognostic variables to be independent predictors of longer OS (Table 2): ECOG PS $0-1(\mathrm{P}=0.001)$, LS $(\mathrm{P}=0.021)$, and positive $\mathrm{SP} 142$ assay $(\mathrm{P}=0.011)$.

\section{Discussion}

PD-L1, which is expressed on many cancer and IC, plays an important role in blocking the cancer immunity cycle by binding $\mathrm{PD}-1$, which is a negative regulator of $\mathrm{T}$ cell activation. Therefore, blocking PD-1/PD-L1 allows T cells to be active and to kill TC (11). Cancer immunotherapy using PD-1/PD-L1 inhibitors, which have been longsought after to unleash the immune system against 
Table 3 The prevalence of PD-L1 expression in studies using FDA-approved PD-L1 assays

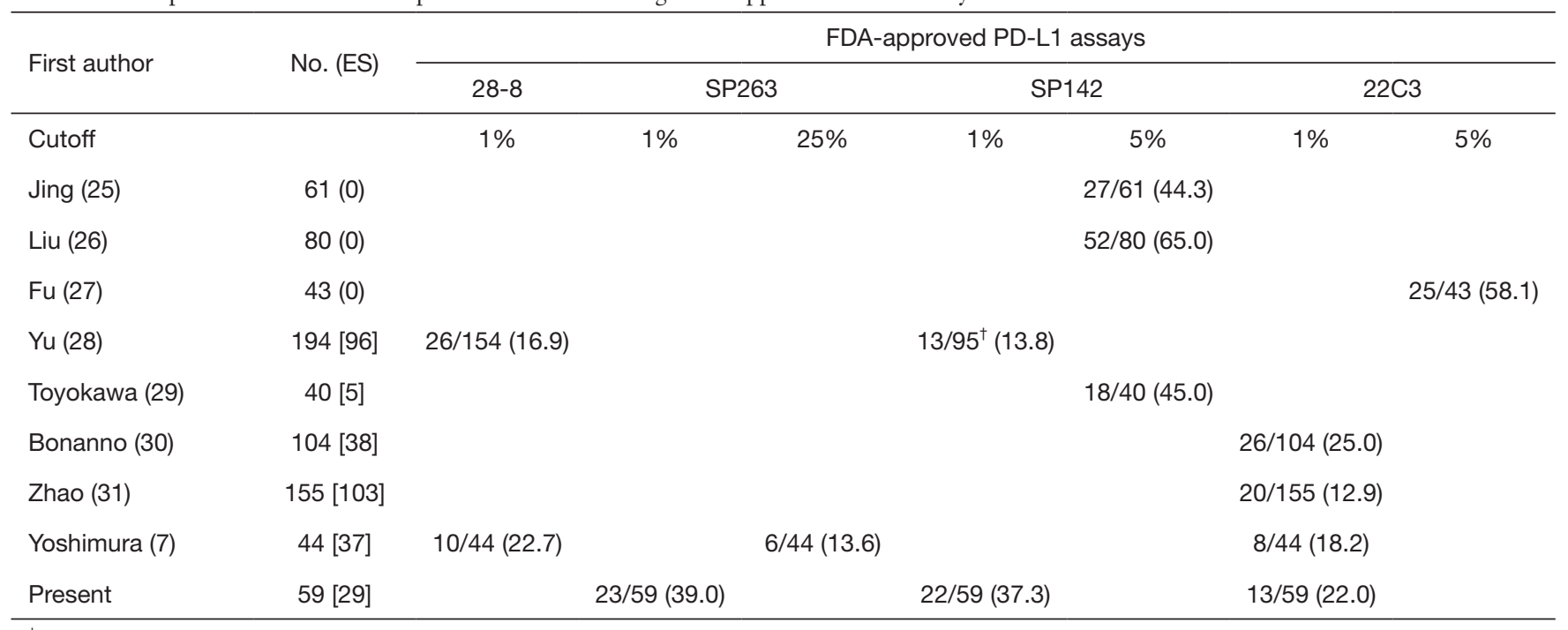

${ }^{\dagger}$, only limited stage patients. ES, extensive stage.

tumors, is a ground-breaking turning point in the field of oncology (3). These PD-1/PD-L1 inhibitors have become an emerging strategy for treatment of multiple cancers, either as first-line treatment or when standard first-line treatment has failed. Unfortunately, the clinical benefits of PD-1/PD-L1 inhibitors only occurs in a minor subset of certain cancers $(2,6,10-12)$. Therefore, it is of importance to identify patients who may potentially benefit from PD-1/PD-L1 inhibitors. Although PD-L1 expression is not a perfect biomarker, PD-L1 expression on TC (or IC) constitutes a logical biomarker for the prediction of treatment response to the PD-1/PD-L1 inhibitors so far (2). However, since the effects of PD-1/PD-L1 inhibitors in SCLC have become known only recently (17), little is known about predictive biomarkers of PD-L1 expression. Therefore, larger studies are urgently needed to study the roles of predictive biomarkers of PD-L1 expression in SCLC.

In the present study, the positivity of three FDAapproved PD-L1 assays, which was defined as PD-L1 expression $\geq 1 \%$ of TC (or IC), was $39.0 \%$ with SP263, $37.3 \%$ with SP142, and $22.0 \%$ with $22 \mathrm{C} 3$. Furthermore, the positive group for $1 / 3$ PD-L1 assays was observed in $31(52.5 \%)$ of the total of 59 patients with SCLC. A recent meta-analysis of PD-L1 expression in SCLC, which included 27 studies enrolling a total of 2,792 patients, reported that the pooled estimate of PD-L1 expression was $26.0 \%$ (19). However, there were large differences in the rate of PD-L1 expression between the studies included, varying from $0 \%$ to $82.8 \%$. The authors also included studies that did not use FDA-approved PD-L1 assays (19). Using two recently published meta-analyses $(18,19)$, we reviewed the prevalence of $\mathrm{PD}-\mathrm{L} 1$ expression in studies using FDA-approved PD-L1 assays in SCLC, which were the same as those used in our study, for a more accurate comparison (Table 3). A total of eight studies used SP142 and/or 22C3 PD-L1 assays (7,25-31); only one study used the SP263 assay (7). As seen in Table 3 showing our results, the prevalence of PD-L1 expression did not show large difference as they did in the meta-analysis and was lower compared to what has been reported for NSCLC $(14,16,19)$. Our study showed that age was correlated with PD-L1 expression in SCLC, similar to a previous study (28). Several studies reported that PD-L1 expression in SCLC is not related to other clinical factors other than stage or age (7,25-30). These results suggest PD-L1 expression in SCLC may become a potent independent prognostic factor. To the best of our knowledge, the present study is the first report on the clinical impact of PD-L1 expression in SCLC using the three most widely used validated PD-L1 assays (SP263, SP142 and 22C3) in clinical practice.

On the other hand, PD-L1 expression was significantly associated with poor OS for patients with several solid tumors, including NSCLC $(14,16)$. Our study showed that positivity for PD-L1, especially 1/3 PD-L1 and SP142 assays, was statistically significantly associated with longer OS in SCLC. Although the other PD-L1 assays (SP263 and 22C3) were not significantly different between positive 
and negative groups, the positive group of PD-L1 assays tended to have longer OS than those with the negative group of PD-L1 assay. A meta-analysis, including several studies showing contradictory results on survival, indicated that PD-L1 expression showed a trend towards longer OS in SCLC patients (19). This result is consistent with our findings. Furthermore, positive SP142 assay was found to be a more significant independent prognostic factor than the NLR, which has been reported as a prognostic factor in patients with various types of cancer $(32,33)$. Interestingly, the survival subgroup analysis of ES patients indicating that positive results of each PD-L1 assay showed more distinct differences than with the analysis of LS patients. These results suggest that the clinical impact of PD-L1 expression in SCLC patients may become a robust prognostic or predictive biomarker in ES than LS patients.

The clinical studies of immunotherapy using ICIs have led to more treatment options in patients with SCLC (34). Based on the results of several clinical studies (17,35-37), PD-1/PD-L1 inhibitors, namely pembrolizumab, nivolumab, atezolizumab, and durvalumab, have been approved by the FDA as the treatment options of first or second and/ or more lines in patients with recurrent or metastatic SCLC. Furthermore, the FDA has granted approval for the combination of atezolizumab, carboplatin, and etoposide for the frontline treatment of patients with ES-SCLC based on study findings, which resulted in significantly longer OS in the atezolizumab group than chemotherapy alone (median OS, 12.3 vs. 10.3 months, respectively; hazard ratio, 0.70; $\mathrm{P}=0.007)(17)$. Recently, updated results with a follow-up of 22.9 months continued to demonstrate an improvement in OS with atezolizumab group and a similar safety profile compared with chemotherapy alone group in patients with ES-SCLC (38). Atezolizumab is the first cancer immunotherapy approved for the initial treatment of ES-SCLC, which is especially difficult to treat. SP142 was approved by the FDA as a "complementary diagnostic" test for atezolizumab. The advent of atezolizumab signifies the beginning of a new era in the treatment of patients with ESSCLC. Therefore, the findings of our study, including the positive SP142 assay, is a significant independent predictor of longer OS, which may provide useful information to patients with ES-SCLC and clinicians considering atezolizumab in the foreseeable future.

There are several limitations to our study that should be noted. First, the retrospective nature of this study is associated with limitations that pertain to selection, exclusion, and recall bias. Second, this study is a single- center study with a relatively small sample size. To overcome these limitations, we prospectively assessed the three validated PD-L1 IHC analyses in same tissue samples of each patient at diagnosis. For accurate staging, enrollment was limited to patients staged with contrastenhanced chest CT scans, PET/CT (and/or whole-body bone scan), and brain imaging to maintain the homogeneity of the population. Moreover, we only enrolled patients with histologically diagnosed SCLC in a relatively short period of time (about 27 months) to improve the quality of our study, even though SCLC accounts for about $13 \%$ of all lung cancers. Therefore, we believe that this inclusion criterion determined the size of the sample and improved the study quality. Third, treatment modalities of our study were not uniform, which may have caused inherent heterogeneity in the retrospective study. Therefore, we used the accurate staging based on several imaging studies instead of the heterogeneous treatment modalities as a variable. Finally, all specimens in our study were obtained from biopsy samples, not surgical samples. Biopsy samples are considered to potentially produce a discrepancy in PDL1 expression regarding the tumor volume of SCLC (7). However, it is generally very rare to perform surgical procedures in SCLC patients. A study showed that PD-L1 expression in biopsy samples was similar to that of surgical specimens (39).

\section{Conclusions}

Our results indicate that the prevalence of PD-L1 expression in SCLC, assessed using three validated PD-L1 assays, is lower compared to what has been reported for NSCLC. The expression of each PD-L1 assay is associated with longer OS, and the positive result of the SP142 assay is a particularly significant independent prognostic factor in patients with SCLC. Furthermore, expression of three PD-L1 assays in patients with ES-SCLC is associated with better outcome than LS patients. The clinical impacts of SP142 expression will become more importance in a new era of atezolizumab in ES-SCLC. Although further validation using larger cohorts is required to generalize our results, this information will benefit clinicians and patients in determining the immunotherapy for patients with ES-SCLC.

\section{Acknowledgments}

Funding: This research was supported by Basic Science Research Program through the National Research 
Foundation of Korea (NRF) funded by the Ministry of Education (Grant No. NRF-2018R1D1A1B07050096).

\section{Footnote}

Reporting Checklist: The authors have completed the REMARK reporting checklist. Available at http://dx.doi. org/10.21037/tlcr-21-165

Data Sharing Statement: Available at http://dx.doi. org/10.21037/tlcr-21-165

Peer Review File: Available at http://dx.doi.org/10.21037/ tlcr-21-165

Conflicts of Interest: All authors have completed the ICMJE uniform disclosure form (available at http://dx.doi. org/10.21037/tlcr-21-165). The authors have no conflicts of interest to declare.

Ethical Statement: The authors are accountable for all aspects of the work in ensuring that questions related to the accuracy or integrity of any part of the work are appropriately investigated and resolved. The study was conducted in accordance with the Declaration of Helsinki (as revised in 2013). The study was approved by the Institutional Review Board of INHA UNIVERSITY HOSPITAL (aproval No. 202008032) and informed consent was taken from all individual participants.

Open Access Statement: This is an Open Access article distributed in accordance with the Creative Commons Attribution-NonCommercial-NoDerivs 4.0 International License (CC BY-NC-ND 4.0), which permits the noncommercial replication and distribution of the article with the strict proviso that no changes or edits are made and the original work is properly cited (including links to both the formal publication through the relevant DOI and the license). See: https://creativecommons.org/licenses/by-nc-nd/4.0/.

\section{References}

1. Howlader N, Krapcho M, Miller D, et al. SEER Cancer Statistics Review (CSR), 1975-2016 Bethesda. 2019.

2. Gibney GT, Weiner LM, Atkins MB. Predictive biomarkers for checkpoint inhibitor-based immunotherapy. Lancet Oncol 2016;17:e542-51.

3. Couzin-Frankel J. Breakthrough of the year 2013. Cancer immunotherapy. Science 2013;342:1432-3.

4. Herbst RS, Baas P, Kim DW, et al. Pembrolizumab versus docetaxel for previously treated, PD-L1-positive, advanced non-small-cell lung cancer (KEYNOTE-010): a randomised controlled trial. Lancet 2016;387:1540-50.

5. Fehrenbacher L, Spira A, Ballinger M, et al. Atezolizumab versus docetaxel for patients with previously treated non-small-cell lung cancer (POPLAR): a multicentre, open-label, phase 2 randomised controlled trial. Lancet 2016;387:1837-46.

6. Borghaei H, Paz-Ares L, Horn L, et al. Nivolumab versus Docetaxel in Advanced Nonsquamous Non-Small-Cell Lung Cancer. N Engl J Med 2015;373:1627-39.

7. Yoshimura A, Yamada T, Miyagawa-Hayashino A, et al. Comparing three different anti-PD-L1 antibodies for immunohistochemical evaluation of small cell lung cancer. Lung Cancer 2019;137:108-12.

8. Hendry S, Byrne DJ, Wright GM, et al. Comparison of Four PD-L1 Immunohistochemical Assays in Lung Cancer. J Thorac Oncol 2018;13:367-76.

9. Rimm DL, Han G, Taube JM, et al. A Prospective, Multi-institutional, Pathologist-Based Assessment of 4 Immunohistochemistry Assays for PD-L1 Expression in Non-Small Cell Lung Cancer. JAMA Oncol 2017;3:1051-8.

10. Taube JM, Klein A, Brahmer JR, et al. Association of PD1, PD-1 ligands, and other features of the tumor immune microenvironment with response to anti-PD-1 therapy. Clin Cancer Res 2014;20:5064-74.

11. Herbst RS, Soria JC, Kowanetz M, et al. Predictive correlates of response to the anti-PD-L1 antibody MPDL3280A in cancer patients. Nature 2014;515:563-7.

12. Topalian SL, Hodi FS, Brahmer JR, et al. Safety, activity, and immune correlates of anti-PD-1 antibody in cancer. $\mathrm{N}$ Engl J Med 2012;366:2443-54.

13. Garon EB, Rizvi NA, Hui R, et al. Pembrolizumab for the treatment of non-small-cell lung cancer. N Engl J Med 2015;372:2018-28.

14. Li H, Xu Y, Wan B, et al. The clinicopathological and prognostic significance of PD-L1 expression assessed by immunohistochemistry in lung cancer: a meta-analysis of 50 studies with 11,383 patients. Transl Lung Cancer Res 2019;8:429-49.

15. Zhang M, Li G, Wang Y, et al. PD-L1 expression in lung cancer and its correlation with driver mutations: a metaanalysis. Sci Rep 2017;7:10255.

16. Wu P, Wu D, Li L, et al. PD-L1 and Survival in Solid Tumors: A Meta-Analysis. PLoS One 2015;10:e0131403.

17. Horn L, Mansfield AS, Szczesna A, et al. First-Line 
Atezolizumab plus Chemotherapy in Extensive-Stage Small-Cell Lung Cancer. N Engl J Med 2018;379:2220-9.

18. Cai H, Zhang H, Jiang Y. Prognostic and Clinicopathological Value of Programmed Cell Death Ligand1 Expression in Patients With Small Cell Lung Cancer: A Meta-Analysis. Front Oncol 2020;10:1079.

19. Acheampong E, Abed A, Morici M, et al. Tumour PD-L1 expression in small-cell lung cancer: A systematic review and meta-analysis. Cells 2020;9:2393.

20. Goldstraw P, Chansky K, Crowley J, et al. The IASLC Lung Cancer Staging Project: Proposals for Revision of the TNM Stage Groupings in the Forthcoming (Eighth) Edition of the TNM Classification for Lung Cancer. J Thorac Oncol 2016;11:39-51.

21. Tsimafeyeu I, Imyanitov E, Zavalishina L, et al. Agreement between PDL1 immunohistochemistry assays and polymerase chain reaction in non-small cell lung cancer: CLOVER comparison study. Sci Rep 2020;10:3928.

22. Chowdhury MZI, Turin TC. Variable selection strategies and its importance in clinical prediction modelling. Fam Med Community Health 2020;8:e00262.

23. Hong HG, Zheng Q, Li Y. Forward regression for Cox models with high-dimensional covariates. J Multivar Anal 2019;173:268-90.

24. Akoglu H. User's guide to correlation coefficients. Turk J Emerg Med 2018;18:91-3.

25. Jing $W$, Wang S, Ding X, et al. High level of programmed death ligand 1 (PD-L1) predicts longer survival in patients with resectable small cell lung cancer. Int J Clin Exp Pathol 2018;11:2675-82.

26. Liu J, Lu Z, Wang W, et al. Programmed death-ligand 1 positivity can predict improved survival and a lower risk of brain metastasis in patients with resectable small cell lung cancer. Oncol Lett 2018;16:2373-81.

27. Fu X, Liu Z, Xiang L, et al. PD-L1 Predicts Poor Prognosis in Surgically Resected Limited Stage Small-Cell Lung Cancer. Cancer Manag Res 2020;12:10939-48.

28. Yu H, Batenchuk C, Badzio A, et al. PD-L1 Expression by Two Complementary Diagnostic Assays and mRNA In Situ Hybridization in Small Cell Lung Cancer. J Thorac Oncol 2017;12:110-20.

29. Toyokawa G, Takada K, Haratake N, et al. Favorable Disease-free Survival Associated with Programmed Death Ligand 1 Expression in Patients with Surgically Resected Small-cell Lung Cancer. Anticancer Res 2016;36:4329-36.

30. Bonanno L, Pavan A, Dieci MV, et al. The role of immune microenvironment in small-cell lung cancer: Distribution of PD-L1 expression and prognostic role of FOXP3positive tumour infiltrating lymphocytes. Eur J Cancer 2018;101:191-200.

31. Zhao X, Kallakury B, Chahine JJ, et al. Surgical Resection of SCLC: Prognostic Factors and the Tumor Microenvironment. J Thorac Oncol 2019;14:914-23.

32. Jeong MJ, Park JH, Hur SY, et al. Preoperative Neutrophil-to-Lymphocyte Ratio as a Prognostic Factor in Uterine Sarcoma. J Clin Med 2020;9:2898.

33. Lee YS, Nam HS, Lim JH, et al. Prognostic impact of a new score using neutrophil-to-lymphocyte ratios in the serum and malignant pleural effusion in lung cancer patients. BMC Cancer 2017; 17:557.

34. Network. NCC. NCCN clinical practice guidelines in oncology (NCCN guidelines). Small cell lung cancer (Version 3.2021). Available online: https://www.nccn. org/guidelines/guidelines-detail? category=1\&id=1462. Accessed March 23, 2021.

35. Paz-Ares L, Dvorkin M, Chen Y, et al. Durvalumab plus platinum-etoposide versus platinum-etoposide in firstline treatment of extensive-stage small-cell lung cancer (CASPIAN): a randomised, controlled, open-label, phase 3 trial. Lancet 2019;394:1929-39.

36. Chung HC, Lopez-Martin JA, Kao SC-H, et al. Phase 2 study of pembrolizumab in advanced small-cell lung cancer (SCLC): KEYNOTE-158. J Clin Oncol 2018;36:8506.

37. Ott PA, Elez E, Hiret S, et al. Pembrolizumab in Patients With Extensive-Stage Small-Cell Lung Cancer: Results From the Phase Ib KEYNOTE-028 Study. J Clin Oncol 2017;35:3823-9.

38. Liu SV, Reck M, Mansfield AS, et al. Updated Overall Survival and PD-L1 Subgroup Analysis of Patients With Extensive-Stage Small-Cell Lung Cancer Treated With Atezolizumab, Carboplatin, and Etoposide (IMpower133). J Clin Oncol 2021;39:619-30.

39. Heymann JJ, Bulman WA, Swinarski D, et al. PD-L1 expression in non-small cell lung carcinoma: Comparison among cytology, small biopsy, and surgical resection specimens. Cancer Cytopathol 2017;125:896-907.

Cite this article as: Lee YS, Lim JH, Ryu W, Park MH, Kim L, Kim K, Kim WY, Nam HS. The clinical impact of three validated PD-L1 immunohistochemistry assays as a prognostic factor in small cell lung cancer. Transl Lung Cancer Res 2021;10(6):2539-2550. doi: 10.21037/tlcr-21-165 
Table S1 Baseline characteristics according to the positive of each PD-L1 assays

\begin{tabular}{|c|c|c|c|c|c|c|c|c|c|c|}
\hline \multirow{3}{*}{ Variables } & \multirow{3}{*}{ No. } & \multicolumn{9}{|c|}{ Three Validated PD-L1 Immunohistochemistry Assays } \\
\hline & & \multicolumn{3}{|c|}{ SP263 Expression } & \multicolumn{3}{|c|}{ SP142 Expression } & \multicolumn{3}{|c|}{ 22C3 Expression } \\
\hline & & $<1 \%$ & $\geq 1 \%$ & $\mathrm{p}$ & $<1 \%$ & $\geq 1 \%$ & $\mathrm{p}$ & $<1 \%$ & $\geq 1 \%$ & $\mathrm{p}$ \\
\hline Age & & & & 0.043 & & & 0.019 & & & 0.747 \\
\hline$<70$ & 34 & 17 & 17 & & 17 & 17 & & 26 & 8 & \\
\hline$\geq 70$ & 25 & 19 & 6 & & 20 & 5 & & 20 & 5 & \\
\hline Sex & & & & 0.072 & & & 0.396 & & & 0.999 \\
\hline Male & 53 & 30 & 23 & & 32 & 21 & & 41 & 12 & \\
\hline Female & 6 & 6 & 0 & & 5 & 1 & & 5 & 1 & \\
\hline PS & & & & 0.174 & & & 0.184 & & & 0.426 \\
\hline $0-1$ & 48 & 27 & 21 & & 28 & 20 & & 36 & 12 & \\
\hline $2-4$ & 11 & 9 & 2 & & 9 & 2 & & 10 & 1 & \\
\hline Smoking & & & & 0.274 & & & 0.286 & & & 0.999 \\
\hline Current+Former & 56 & 33 & 23 & & 34 & 22 & & 43 & 13 & \\
\hline Never & 3 & 3 & 0 & & 3 & 0 & & 3 & 0 & \\
\hline NLR & & & & 0.508 & & & 0.641 & & & 0.754 \\
\hline$<2.5$ & 21 & 14 & 7 & & 14 & 7 & & 17 & 4 & \\
\hline$\geq 2.5$ & 38 & 22 & 16 & & 23 & 15 & & 29 & 9 & \\
\hline $\mathrm{CEA}(\mathrm{ng} / \mathrm{ml})^{\dagger}$ & & & & 0.597 & & & 0.999 & & & 0.202 \\
\hline $0-5.2$ & 11 & 5 & 6 & & 6 & 5 & & 10 & 1 & \\
\hline$>5.2$ & 18 & 10 & 8 & & 10 & 8 & & 12 & 6 & \\
\hline $\mathrm{LDH}(\mathrm{IU} / \mathrm{L})^{\dagger}$ & & & & 0.883 & & & 0.732 & & & 0.867 \\
\hline $0-250$ & 21 & 12 & 9 & & 13 & 8 & & 16 & 5 & \\
\hline$>250$ & 27 & 16 & 11 & & 18 & 9 & & 20 & 7 & \\
\hline T stage & & & & 0.555 & & & 0.181 & & & 0.735 \\
\hline $1-2$ & 18 & 12 & 6 & & 9 & 9 & & 15 & 3 & \\
\hline $3-4$ & 41 & 24 & 17 & & 28 & 13 & & 31 & 10 & \\
\hline $\mathrm{N}$ stage & & & & 0.490 & & & 0.152 & & & 0.676 \\
\hline $0-1$ & 10 & 5 & 5 & & 4 & 6 & & 7 & 3 & \\
\hline $2-3$ & 49 & 31 & 18 & & 33 & 16 & & 39 & 10 & \\
\hline Stage & & & & 0.486 & & & 0.040 & & & 0.701 \\
\hline Limited (LS) & 30 & 17 & 13 & & 15 & 15 & & 24 & 6 & \\
\hline Extensive (ES) & 29 & 19 & 10 & & 22 & 7 & & 22 & 7 & \\
\hline Brain meta & & & & 0.999 & & & 0.999 & & & 0.398 \\
\hline No & 50 & 30 & 20 & & 31 & 19 & & 40 & 10 & \\
\hline Yes & 9 & 6 & 3 & & 6 & 3 & & 6 & 3 & \\
\hline SP263 & & & & & & & $<0.001$ & & & $<0.001$ \\
\hline Negative & 36 & & & & 30 & 6 & & 34 & 2 & \\
\hline Positive & 23 & & & & 7 & 16 & & 12 & 11 & \\
\hline SP142 & & & & $<0.001$ & & & & & & 0.010 \\
\hline Negative & 37 & 30 & 7 & & & & & 33 & 4 & \\
\hline Positive & 22 & 6 & 16 & & & & & 13 & 9 & \\
\hline $22 \mathrm{C} 3$ & & & & $<0.001$ & & & 0.010 & & & \\
\hline Negative & 46 & 34 & 12 & & 33 & 13 & & & & \\
\hline Positive & 13 & 2 & 11 & & 4 & 9 & & & & \\
\hline
\end{tabular}

${ }^{\dagger}$ Dichotomized by cutoff of normal value. CEA, carcinoembryonic antigen; ECOG PS, Eastern Cooperative Oncology Group performance status; LDH, lactate dehydrogenase; NLR, neutrophi//ymphocyte ratio. 

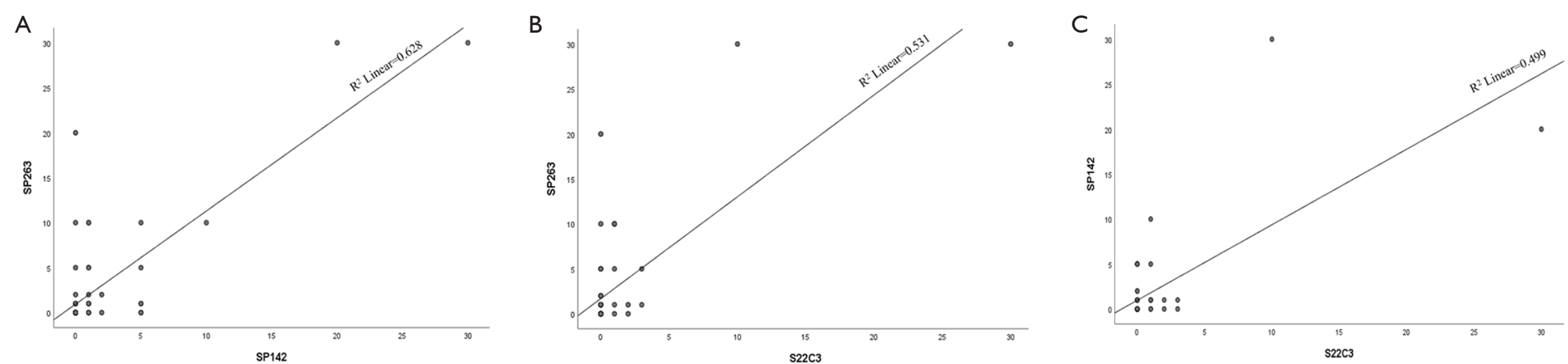

Figure S1 Correlation of PD-L1 expression using the three PD-L1 assays. Scatter diagrams illustrating the correlation between expression levels according to (A) PD-L1 SP263 and SP142 assays, (B) PD-L1 SP263 and 22C3 assays, and (C) PD-L1 SP142 and 22C3 assays.
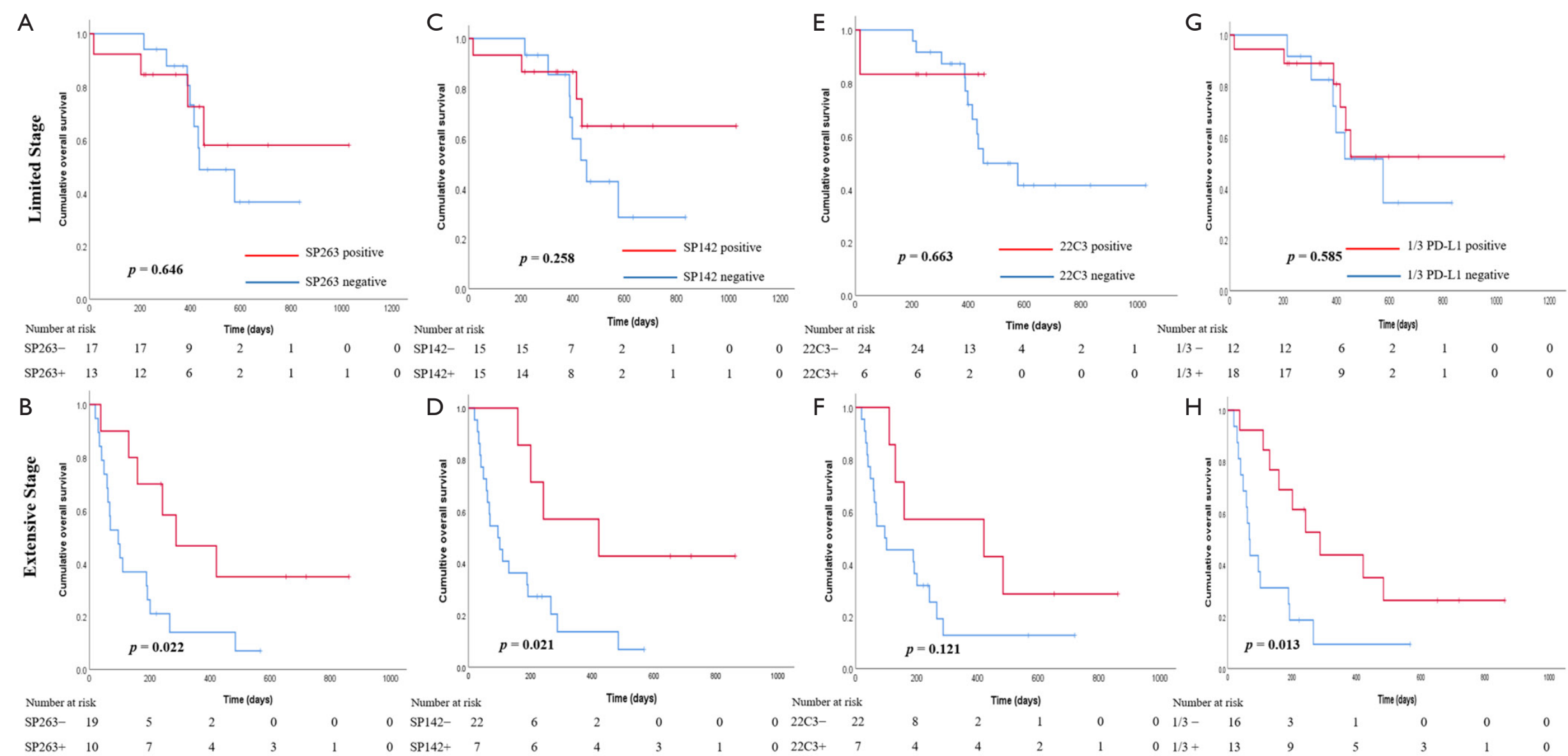

Figure S2 Kaplan-Meier curves of overall survival according to PD-L1 expression status by SCLC stage. (A, B) PD-L1 SP263 in limited-stage (LS) and extensive-stage (ES), (C, D) PD-L1 SP142 in LS and ES, (E, F) PD-L1 IHC 22C3 in LS and ES, (G, H) positive results for at least one of the three PD-L1 assays (1/3 PD-L1) in LS and ES. 